\title{
Effect of the spin-orbit interaction on the band gap of half metals
}

\author{
Ph. Mavropoulos, K. Sato, R. Zeller, and P. H. Dederichs \\ Institut für Festkörperforschung, Forschungszentrum Jülich, D-52425 Jülich, Germany
}

V. Popescu and H. Ebert

Department Chemie/Physikalische Chemie, University of Munich, Butenandtstr. 5-13, D-81377 Munich, Germany

(Received 7 August 2003; revised manuscript received 18 November 2003; published 26 February 2004)

\begin{abstract}
The spin-orbit interaction can cause a nonvanishing density of states (DOS) within the minority-spin band gap of half metals around the Fermi level. We examine the magnitude of the effect in Heusler alloys, zincblende half metals, and diluted magnetic semiconductors, using first-principles calculations. We find that the ratio of spin-down to spin-up DOS at the Fermi level can range from below $1 \%$ (e.g., $0.5 \%$ for NiMnSb) over several percents $\left[4.2 \%\right.$ for $(\mathrm{Ga}, \mathrm{Mn}) \mathrm{As}$ ] to $13 \%$ for $\mathrm{MnBi}$. This gives a spin polarization $P$ at $E_{F}$ ranging from above $99 \%$ for $\mathrm{NiMnSb}$ to $P \approx 92 \%$ for $(\mathrm{Ga}, \mathrm{Mn})$ As and to $P \approx 77 \%$ for $\mathrm{MnBi}$.
\end{abstract}

DOI: 10.1103/PhysRevB.69.054424

PACS number(s): 75.50.Cc, 71.20.-b, 75.30. $-\mathrm{m}$

Half metals are materials showing spin ferromagnetism, but with the exotic property of presenting a metallic density of states (DOS) only for the one spin direction (usually majority), in parallel with a clear band gap around the Fermi level $E_{F}$ for the other direction. The first of the kind to be reported were half metallic Heusler alloys by de Groot et al. in $1983 .{ }^{1}$ More recently, other half metals were found, such as $\mathrm{CrO}_{2},{ }^{2} \quad \mathrm{La}_{0.7} \mathrm{Sr}_{0.3} \mathrm{MnO}_{3},{ }^{2}$ or diluted magnetic semiconductors. ${ }^{3}$ Furthermore, ordered zinc-blende CrAs and $\mathrm{CrSb}$ were fabricated by molecular beam epitaxy ${ }^{4}$ and relevant calculations suggest these and many similar zincblende systems to be half metallic. ${ }^{5-7}$ So has been the case with a variety of Heusler alloys. ${ }^{8}$ The rising interest in half metallic systems is partly caused by their potential applications in spin electronics, ${ }^{9}$ since junctions made of half metals should ideally present infinite magnetoresistance ratio or $100 \%$ spin polarized currents.

Although the $a b$ initio calculations show the presence of half metallicity in this variety of materials, with a clear band gap around $E_{F}$ for the minority spin, this can be partly suppressed by, e.g., defects, ${ }^{10}$ spin excitations at increased temperature, or non-quasiparticle states. ${ }^{11}$ Even at low temperature and in the defect-free case, there is still the question of spin-orbit interaction. Although this is weak and in many cases can be neglected, in principle it should couple the two spin channels so that the presence of states around $E_{F}$ for the majority spin is partly reflected in the minority DOS. Thus one cannot have a real gap, but a small DOS, its smallness depending on the strength of the spin-orbit coupling for each material; this effect could have also implications in the ideas about spin-dependent transport and spintronics devices. It is the purpose of this paper to give a quantitative picture for this effect for a few typical systems, and to argue if and how crucially this could affect spin-dependent transport. As model systems we have chosen the Heusler alloy NiMnSb, the ordered zinc-blende alloys $\mathrm{CrAs}, \mathrm{CrSb}$ and $\mathrm{MnBi}$, and the chemical disorder in diluted magnetic semiconductor $\mathrm{Ga}_{0.95} \mathrm{Mn}_{0.05}$ As.

Our first-principles calculations are based on the screened Korringa-Kohn-Rostoker Green function method within the local density approximation, incorporating fully relativistic effects (from where the spin-orbit interaction emerges). For the description of the diluted magnetic semiconductors, the coherent potential approximation (CPA) was also employed. Details about the method can be found in Ref. 12. In the calculations we have used the atomic sphere approximation, in which it is assumed that the potential around each atomic site is spherically symmetric. In the open structures, such as the zinc-blende, the large empty interstitial space is accounted for by placing at appropriate positions empty spheres (treated as atomic spheres with no nuclear charge). In the expansion of the Green function in local orbitals a cutoff of $l_{\max }=3$ was taken for the ordered compounds and of $l_{\max }=2$ for the CPA calculations. We have employed both the fully relativistic (solving the Dirac equation) and the scalar relativistic ${ }^{13}$ methods for comparison. The latter includes all other relativistic effects except spin-orbit coupling, so that the two spin channels are decoupled and half metals appear with a clean gap for spin-down around $E_{F}$.

The DOS $n_{\sigma}(E)$ per spin $\sigma$ is related to the retarded Green function $G_{\sigma}\left(\vec{r}, \overrightarrow{r^{\prime}} ; E\right)$ via

$$
n_{\sigma}(E)=-\frac{1}{\pi} \operatorname{Im} \int G_{\sigma}(\vec{r}, \vec{r} ; E) d^{3} r .
$$

Usually for the calculation a very small imaginary part $i \epsilon$ is added to the real energy $E$ and the Green function and DOS are evaluated at $E+i \epsilon$. In this way the DOS of every eigenstate is no more a $\delta$ function of the energy but is rather smoothed via a Lorentzian broadening. This method has the advantage of needing fewer $\vec{k}$ points for the integration in the Brillouin zone to obtain a smooth DOS, saving computational time. Nevertheless there is the disadvantage that, in the presence of a band gap, the band edges are not sharp and one also obtains a very low nonzero DOS within the gap due to the Lorentzian broadening. In our case this causes a problem, since the DOS within the minority gap due to spin-orbit coupling is also small and drowned in the background of the Lorentzian broadening. To overcome this difficulty one would have to go much closer to the real energy axis, which would demand too many $\vec{k}$ points. We have used an alternative, which saves calculation time: Since the Green function 
is analytical in the complex energy plane $E_{z}$, we are able to extrapolate to the real energy axis by knowing the variation of the Green function for $E_{z}$ along a line parallel to the real axis. To our knowledge, this method was first proposed by Gray and Kaplan, ${ }^{14}$ who also reported on the numerical limitations. The resulting accuracy within the gap is much better than that of the traditional method, and good enough for our purposes, although sometimes numerical fluctuations of the DOS are visible.

In our calculations we solve the Dirac equation, thus treating the spin-orbit term exactly. But for a better understanding we give now a brief description of the first order effect of the interaction in a Schrödinger picture. We remind that the spinorbit coupling of the two spin channels is related to the unperturbed potential $V(r)$ around each atom via the angular momentum operator $\vec{L}$ and the Pauli spin matrix $\vec{\sigma}$ :

$$
V_{\mathrm{so}}(r)=\frac{1}{2 m^{2} c^{2}} \frac{\hbar}{2} \frac{1}{r} \frac{d V}{d r} \vec{L} \cdot \vec{\sigma}=\left(\begin{array}{cc}
V_{\mathrm{so}}^{\uparrow \uparrow} & V_{\mathrm{so}}^{\uparrow \downarrow} \\
V_{\mathrm{so}}^{\downarrow \uparrow} & V_{\mathrm{so}}^{\downarrow \downarrow}
\end{array}\right) .
$$

Analyzed in spinor basis, it takes the $2 \times 2$ matrix form above. We denote the two spin directions with $\uparrow$ and $\downarrow$, the unperturbed crystal Hamiltonian for the two spin directions as $H^{0 \uparrow}$ and $H^{0 \downarrow}$, and the unperturbed Bloch eigenfunctions as $\Psi_{n \vec{k}}^{0 \uparrow}$ and $\Psi_{n \vec{k}}^{0 \downarrow}$. Then the Schrödinger equation for the perturbed wave function $\Psi_{n \vec{k}}=\left(\Psi_{n \vec{k}}^{\uparrow}, \Psi_{n \vec{k}}^{\downarrow}\right)$ reads

$$
\left(\begin{array}{cc}
H^{0 \uparrow}+V_{\text {so }}^{\uparrow \uparrow}-E & V_{\text {so }}^{\uparrow \downarrow} \\
V_{\text {so }}^{\downarrow \uparrow} & H^{0 \downarrow}+V_{\text {so }}^{\downarrow \downarrow}-E
\end{array}\right)\left(\begin{array}{c}
\Psi_{n \vec{k}}^{\uparrow} \\
\Psi_{n \vec{k}}^{\downarrow}
\end{array}\right)=0 .
$$

The potential terms $V_{\mathrm{so}}^{\uparrow \downarrow}$ and $V_{\mathrm{so}}^{\downarrow \uparrow}$ are responsible for flipping the spin. Within the gap region of the half metal, where no spin-down states of the unperturbed Hamiltonian exist, the spin-down solution can be found in first order by solving Eq. (3) for $\Psi_{n \vec{k}}^{(1) \downarrow}$ [the index (1) stands for the first-order solution]. The solution reads formally

$$
\Psi_{n \vec{k}}^{(1) \downarrow}=-\frac{1}{H^{\downarrow}+V_{\mathrm{so}}^{\downarrow \downarrow}-E_{n \vec{k}}^{0 \uparrow}} V_{\mathrm{so}}^{\downarrow \uparrow} \Psi_{n \vec{k}}^{0 \uparrow}
$$

with $E_{n \vec{k}}^{0 \uparrow}$ the eigenenergy of the state $\Psi_{n \vec{k}}^{0 \uparrow}$. We see that in the gap region the spin-down intensity is a weak image of the band structure $E_{n \vec{k}}^{0 \uparrow}$ of the spin-up band. Since the DOS is related to $\left|\Psi_{n k}^{(1)}\right|^{2}$, it is expected that within the gap the DOS has a quadratic dependence on the spin-orbit coupling strength: $n_{\downarrow}(E) \sim\left(V_{\text {so }}^{\downarrow \uparrow}\right)^{2}$. But there is also a modification by the term $-1 /\left(H^{\downarrow}+V_{\mathrm{so}}^{\downarrow \downarrow}-E_{n \vec{k}}^{0 \uparrow}\right.$ ) (this is actually the Green function), which increases the weight of the states close to those $\vec{k}$ points where the unperturbed spin-up and spin-down bands cross, i.e., $E_{n \hat{k}}^{0 \uparrow}=E_{n^{\prime} \vec{k}}^{0 \downarrow}$. To see this we can rewrite Eq. (4) by expanding the Green function in spectral representation

$$
\begin{aligned}
\Psi_{n \vec{k}}^{(1) \downarrow} \downarrow(\vec{r})= & \int d^{3} r^{\prime} \sum_{n^{\prime}} \frac{\Psi_{n^{\prime} \vec{k}}^{(0) \downarrow}(\vec{r}) \Psi_{n^{\prime} \cdot \vec{k}}^{(0) \downarrow}\left(\overrightarrow{r^{\prime}}\right)}{E_{n \vec{k}}^{0 \uparrow}-E_{n^{\prime} \vec{k}}^{0 \downarrow}} \\
& \times V_{\mathrm{so}}^{\downarrow \uparrow}\left(\overrightarrow{r^{\prime}}\right) \Psi_{n \vec{k}}^{(0) \uparrow}\left(\overrightarrow{r^{\prime}}\right) \\
= & \sum_{n^{\prime}} \frac{\left\langle\Psi_{n^{\prime} \vec{k}}^{0 \downarrow}\left|V_{\mathrm{so}}^{\downarrow \uparrow}\right| \Psi_{n \vec{k}}^{0 \uparrow}\right\rangle}{E_{n \vec{k}}^{0 \uparrow}-E_{n^{\prime} \vec{k}}^{0 \downarrow}} \Psi_{n^{\prime} \vec{k}}^{0 \downarrow}(\vec{r}) .
\end{aligned}
$$

Here, the summation runs only over the band index $n^{\prime}$ and not over the Bloch vectors $\vec{k}^{\prime}$, because Bloch functions with $\vec{k}^{\prime} \neq \vec{k}$ are mutually orthogonal. Close to the crossing point $E_{n \vec{k}}^{0 \uparrow}-E_{n^{\prime} \vec{k}}^{0 \downarrow}$ the denominator becomes small and the bands strongly couple. Then one should also consider higher orders in the perturbation expansion. Since at the gap edges there exist spin-down bands of the unperturbed Hamiltonian, this effect can become important near the gap edges.

As a measure of the strength of half metallicity we consider the ratio of the minority to the majority DOS at $E_{F}$, $n_{\downarrow}\left(E_{F}\right) / n_{\uparrow}\left(E_{F}\right)$, which should be exactly zero in an ideal half metal, and the spin polarization at $E_{F}$,

$$
\begin{aligned}
P & =\frac{n_{\uparrow}\left(E_{F}\right)-n_{\downarrow}\left(E_{F}\right)}{n_{\uparrow}\left(E_{F}\right)+n_{\downarrow}\left(E_{F}\right)} \\
& \approx 1-2 n_{\downarrow}\left(E_{F}\right) / n_{\uparrow}\left(E_{F}\right) \text { for small } n_{\downarrow} / n_{\uparrow},
\end{aligned}
$$

which should be $100 \%$ in the ideal case.

Now we proceed to the analysis of our results. The results for the Heusler alloy NiMnSb, which was found by de Groot et al. in 1983 to be half metallic, ${ }^{1}$ are shown in Fig. 1. In the top panel, the atom-resolved local DOS (LDOS) is shown, following a scalar relativistic calculation. The Fermi level is within the band gap for minority spin and the material is half metallic. In this scale, the relativistic calculation shows no difference in the DOS compared to the scalar relativistic. In the middle panel the gap region is shown in a relativistic calculation. Again, no minority DOS is seen in this scale. But when we magnify the minority DOS in the lower panel, it becomes clear that the DOS is nonzero in the gap region-it is of the order of $0.5 \%$ of the majority DOS in the vicinity of $E_{F}$, yielding $P \approx 99 \%$. We have checked the DOS in this scale for the scalar relativistic calculation and have found it to be zero, thus we conclude that we see here a spin-orbit effect and not an artificial Lorentzian broadening due to the imaginary part of the energy in the calculation. The fluctuations of the Mn DOS (full line) are due to numerical noise of the $\vec{k}$ integration and extrapolation scheme, and mark its limit of accuracy.

The next example is the ordered half metallic alloy CrAs in the zinc-blende structure. This was invented by Akinaga and co-workers ${ }^{4}$ in 2000 and found to be half metallic. Our results are presented in Fig. 2. In the top panel, the scalar relativistic atom-resolved DOS is shown. The Fermi level is within the minority-spin gap, close to the conduction band edge. (The latter is mainly of $d$ character, containing the wave functions of the $e_{g}$ subspace. ${ }^{7}$ ) Focusing in the range of the gap, we can see the DOS in more detail in the middle panel in a relativistic calculation. Below $-1 \mathrm{eV}$ one recog- 


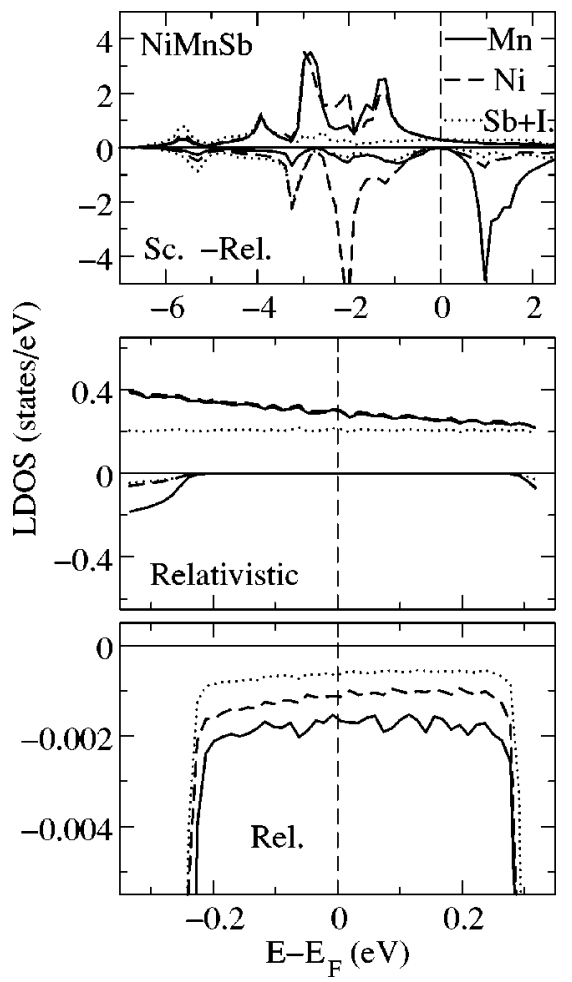

FIG. 1. DOS of NiMnSb. Top: scalar relativistic (I. refers to interstitial volume of the empty sphere). Middle: fully relativistic, focused in the minority-gap region around $E_{F}$. In this scale, the spin-orbit effect is not visible. Bottom: fully relativistic, focused on the minority states within the gap. The fluctuations reflect the numerical accuracy of the method (see text). Around $E_{F}$, the spindown DOS is of the order of $0.5 \%$ of the spin-up DOS, giving $P$ $\approx 99 \%$. Close to the gap edges, the spin-orbit induced minority DOS increases strongly as explained in the text. Negative numbers in the DOS axis correspond to minority spin.

nizes the majority $d$ states of $e_{g}$ character. $^{7}$ Around $-0.9 \mathrm{eV}$ the DOS has a minimum, as the $e_{g}$ states end and the $d$ states of $t_{2 g}$ character start, forming wide bands. In this scale no spin-orbit effect is visible in the minority gap. But if we focus further in energy (lower panel) it is clear that the structure of the majority DOS is reflected in the minority DOS: one can recognize the peak of the $e_{g}$ states below $-1 \mathrm{eV}$, then the minimum and finally the presence of the $t_{2 g}$ band above $-0.8 \mathrm{eV}$. This is the effect of the term $V_{\mathrm{so}}^{\downarrow \uparrow} \Psi_{n \vec{k}}^{\uparrow}$ in Eq. (4). But we also observe the rapid increase of the DOS as the energy approaches the band edges; this comes from the term $-1 /\left(H^{\downarrow}+V_{\text {so }}^{\downarrow \downarrow}-E_{n \vec{k}}^{0 \uparrow}\right)$. The minority DOS in the middle of the gap is again small, giving a minority/majority DOS ratio of $0.2 \%$, whence $P=99.6 \%$. In a similar calculation for zinc-blende CrSb (first fabricated by Zhao et al. ${ }^{15}$ and calculated by $\mathrm{Liu}^{6}$ ) we find that the minority DOS in the middle of the gap is around $0.7 \%$ of its majority counterpart, and $P$ $=98.6 \%$. This rise is expected, as the spin-orbit interaction is stronger for the $5 p$ states of $\mathrm{Sb}$ than for the $4 p$ of As. (The trend can be seen, for example, in a systematic study of the spin-orbit cross section of $s p$ impurities in Mg in Ref. 16, where $\mathrm{Sb}$ is found to have four times higher spin-orbit cross

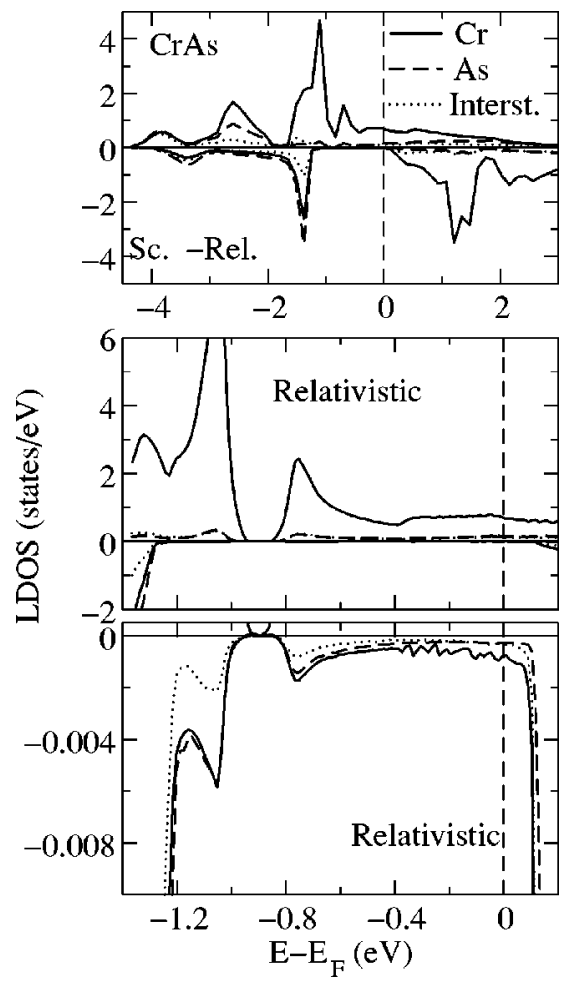

FIG. 2. DOS of zinc-blende CrAs. Top: scalar relativistic. Middle: fully relativistic, focused in the minority-gap region around $E_{F}$. In this scale, the spin-orbit effect is not visible. Bottom: fully relativistic, focused on the minority states within the gap. The reflection of spin-up into spin-down DOS is clear at the peaks at $-1 \mathrm{eV}$ and $-0.8 \mathrm{eV}$. In the middle of the gap, the spin-down DOS is of the order of $0.2 \%$ of the spin-up DOS, i.e., $P \approx 99.6 \%$. Negative numbers in the DOS axis correspond to minority spin.

section than As. Since the spin-orbit interaction is in first approximation an atomic property, the trend is similar here.)

A recent theoretical investigation ${ }^{17}$ has suggested the ordered zinc-blende alloy $\mathrm{MnBi}$ as a good candidate for half metallicity. Although this has not yet been fabricated, we take it as a good example to show the spin-orbit effect, since $\mathrm{Bi}$ is a heavy element with strong spin-orbit interaction because of the $6 \mathrm{p}$ states. The scalar and fully relativistic DOS are shown in Fig. 3. It is evident that here the spin-orbit interaction has a much stronger influence. The minority DOS at $E_{F}$ is of the order of $13 \%$ of its majority counterpart, a huge quantity in comparison to the analogous percentage in CrAs or NiMnSb; the spin polarization at $E_{F}$ is $P=77 \%$. The relativistic effect shows up also in the orbital magnetic moment, being $0.11 \mu_{B}$ and mostly concentrated at the $\mathrm{Mn}$ atom, while the spin moment remains at $4.0 \mu_{B}$ per unit cell as in the scalar and nonrelativistic case. We note that, although the spin-orbit interaction is strong here, the majority spin is still dominating at $E_{F}$. Thus MnBi would be a useful material for spin electronics despite the spin-orbit effect, as its spin polarization at $E_{F}$ is robust against moderate volume change as pointed out in Ref. 17.

As a final example we consider the diluted magnetic semiconductor (DMS) $\mathrm{Ga}_{0.95} \mathrm{Mn}_{0.05}$ As. This material, together with other similar DMS, has been extensively studied 


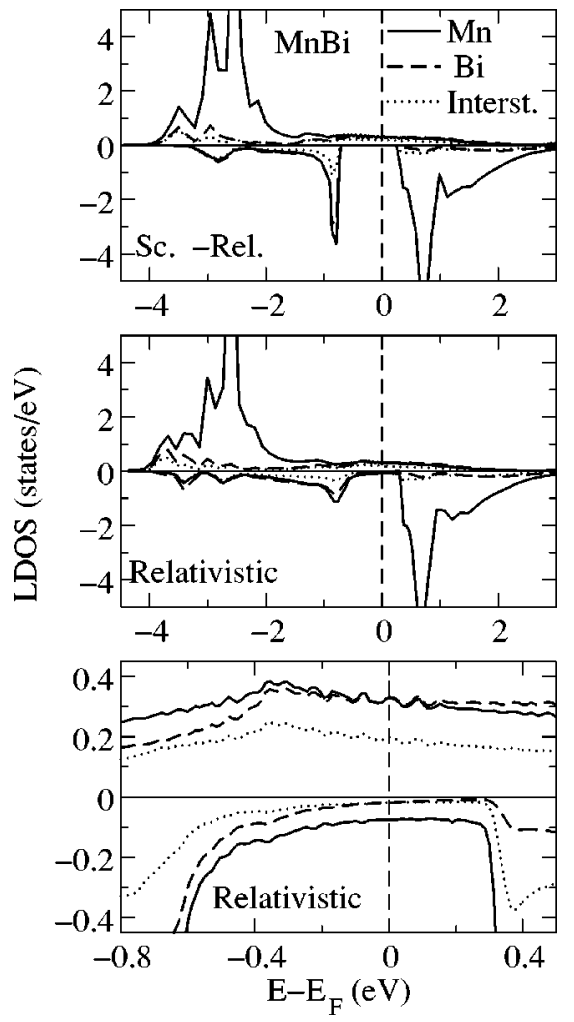

FIG. 3. DOS of zinc-blende MnBi. Top: scalar relativistic. Middle: Fully relativistic. Bottom: fully relativistic, focused on the states within the minority gap. At $E_{F}$, the spin-down DOS is of the order of $13 \%$ of the spin-up DOS. This higher value is expected since the $6 p$ valence states of Bi result in a strong spin-orbit effect. Negative numbers in the DOS axis correspond to minority spin.

in recent years, because it presents ferromagnetism at such low Mn concentrations (discovered by $\mathrm{Ohno}^{3}$ in 1998), so that it is a ferromagnetic semiconductor. In the ideal case, in which the material is calculated to be half metallic, the Mn impurities substitute $\mathrm{Ga}$ atoms in the GaAs lattice. Then the Mn $3 d$ states of the $t_{2 g}$ representation $\left(d_{x y}, d_{y z}\right.$, and $\left.d_{x z}\right)$ hybridize with the $p$ states of the As neighbors, forming bonding and antibonding $p-d$ hybrids and from them states within the GaAs gap for the spin-up direction. Thus the alloy becomes half metallic with $E_{F}$ close to the valence-band edge. Although there is no consensus yet on the physical origin of the ferromagnetic ordering, which seems to depend on the energetic position of the Mn $d$ states, ${ }^{18}$ we have proceeded using the local-density approximation (LDA) result. The density of states is presented in Fig. 4, together with the DMS $(\mathrm{Ga}, \mathrm{Mn}) \mathrm{N}$.

We find that the spin-down DOS at $E_{F}$ is $4.2 \%$ of its spin-up counterpart if we include spin-orbit effects, giving $P=91.9 \%$ while the system is half metallic in the nonrelativistic solution. To understand this relatively high percentage of spin-down states, we observe that $E_{F}$ is very close to the valence-band edge. There, the $p$ states of As give an important spin-orbit effect. This is known to split the valence-band edge of GaAs in two subspaces, one with total (spin + orbital) angular momentum $j=3 / 2$ and one with $j$ $=1 / 2$, so that the valence-band edge has no pure spin
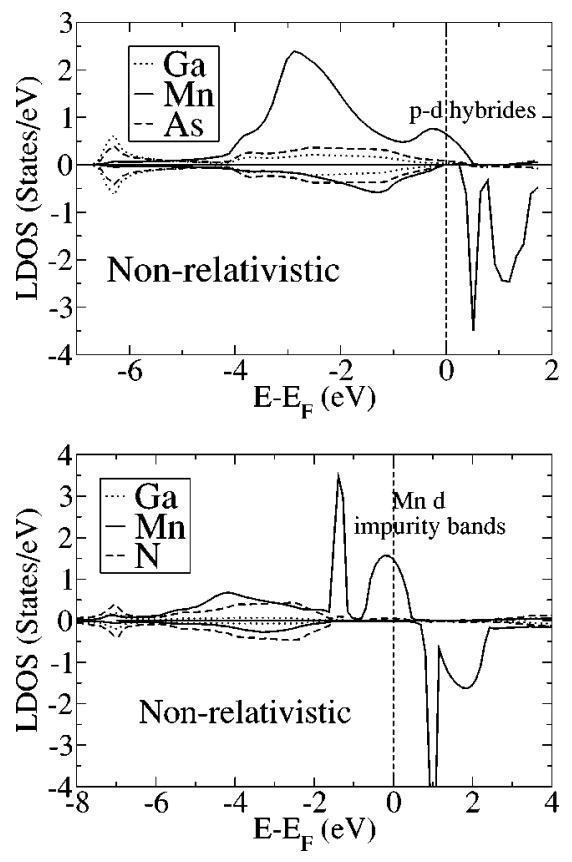

FIG. 4. Up: Local DOS of the diluted magnetic semiconductor $\mathrm{Ga}_{0.95} \mathrm{Mn}_{0.05}$ As. In a nonrelativistic calculation it is a half metal, but when spin-orbit effects are accounted for, the spin-down DOS at $E_{F}$ becomes $4.2 \%$ of the spin-up DOS, since $E_{F}$ is close to the GaAs valence-band edge. Down: Local DOS of the diluted magnetic semiconductor $\mathrm{Ga}_{0.95} \mathrm{Mn}_{0.05} \mathrm{~N}$ in the zinc-blende structure. Contrary to $(\mathrm{Ga}, \mathrm{Mn}) \mathrm{As}$, the Fermi level is now near the middle of the gap, within a Mn impurity $d$ band. This, and the fact that $\mathrm{N}$ is a light element, lead to negligible spin-orbit coupling and minority DOS at $E_{F}$.

character. ${ }^{19}$ In fact, it has been proposed that the value of $P$ depends on a competition between the magnitude of the exchange splitting and the spin-orbit splitting of the valenceband edge. ${ }^{20}$

A recent paper ${ }^{21}$ reports direct measurements of the spin polarization in $(\mathrm{Ga}, \mathrm{Mn}) \mathrm{As}$ via Andreev reflection spectroscopy, giving a value of $P=85 \%$ or $P=90 \%$, depending on the sample. Our calculated value of $P=91.9 \%$ agrees reasonably with these measured data. However, this agreement could be fortuitous, since, on the one hand, the theoretical value could change if the $\mathrm{Mn} d$ states were deeper in energy (a possibility if one goes beyond the LDA), and, on the other hand, the measured values depend also on the exact form of the ferromagnet/superconductor interface, plus the presence of other defects within the sample.

Similar calculations on Mn-doped ferromagnetic zincblende $\mathrm{GaN}$ gave us no spin-orbit effect at $E_{F}$ within numerical accuracy. The difference of $(\mathrm{Ga}, \mathrm{Mn}) \mathrm{N}$ to $(\mathrm{Ga}, \mathrm{Mn}) \mathrm{As}$ is that the $2 p$ valence states of $\mathrm{N}$ compared to the $4 p$ of As (i) have a much lower spin-orbit interaction, and (ii) are lower in energy so that the Mn impurity $d$ band is fully within the band gap, away from the nitrogen-dominated valence-band states, and the spin-orbit effect weakens.

In summary, we have performed first-principles calculations in order to investigate the effect of the spin-orbit coupling to the spin-down band gap of half metallic systems. As typical half-metallic systems, describable by the LDA, we 
have chosen the Heusler alloy NiMnSb, the ordered zincblende alloys $\mathrm{CrAs}, \mathrm{CrSb}$, and $\mathrm{MnBi}$, and the diluted magnetic semiconductors $(\mathrm{Ga}, \mathrm{Mn}) \mathrm{As}$ and $(\mathrm{Ga}, \mathrm{Mn}) \mathrm{N}$. We find that the majority-spin states are partly reflected into the minority band gap. The intensity of the DOS for minority electrons in this energy region depends mainly on the strength of the spin-orbit coupling, heavier $s p$ elements resulting in a higher ratio of minority/majority-spin DOS $n_{\downarrow} / n_{\uparrow}$, thus lower spin polarization $P$, at $E_{F}$. Thus we see a trend of increasing $n_{\downarrow} / n_{\uparrow}$ and decreasing $P$ as we go from CrAs $\left(n_{\downarrow} / n_{\uparrow}=0.2 \%, \quad P=99.6 \%\right)$ to $\mathrm{CrSb} \quad\left(n_{\downarrow} / n_{\uparrow}=0.7 \%, P\right.$ $=98.6 \%)$ and finally to $\mathrm{MnBi}\left(n_{\downarrow} / n_{\uparrow}=13 \%, P=77 \%\right)$. In NiMnSb we find $n_{\downarrow} / n_{\uparrow}=0.5 \%$ and $P=99 \%$. Also, it is important how deep the Fermi level lies within the gap, since majority states close to the gap edges are more drastically spin flipped than states deep in the gap. This results in high minority-spin DOS $\left(n_{\downarrow} / n_{\uparrow}=4.2 \%, P=91.9 \%\right)$ for $(\mathrm{Ga}, \mathrm{M}-$ n)As where $E_{F}$ is very close to the valence-band edge. We have also explained these effects within a first-order approximation. For transport applications in spintronics we conclude that even in compounds with strong spin-orbit effect $[\mathrm{MnBi}$ or $(\mathrm{Ga}, \mathrm{Mn}) \mathrm{As}]$ the minority DOS at $E_{F}$ is still dominated by its majority counterpart (even in $\mathrm{MnBi}$ the ratio is only $13 \%$ ), whence it follows that current should be transported practically only by majority states. On the other hand, comparing the value of $P=99 \%$ for NiMnSb with the much lower experimental result ${ }^{2}$ of $P \approx 58 \%$ in Andreev reflection experiments using a superconducting contact (while, in the bulk, experiments show good half metallicity ${ }^{22}$ ), we conclude that for practical applications other effects, such as gap states due to impurities, ${ }^{10}$ stalking faults, or interface and surface states, could be more important to be eliminated by sample improvements.

\section{ACKNOWLEDGMENTS}

This work was supported by the RT Network of Computational Magnetoelectronics (Contract No. RTN1-199900145) of the European Commission. One of us (V.P.) gratefully acknowledges the financial support of the Deutsche Forschungsgemeinschaft within the DFG-Förderprojekt Grant No. FOG 370/2-1 "Ferromagnet-HalbleiterNanostrukturen: Transport, elektrische und magnetische Eigenschaften."
${ }^{1}$ R.A. de Groot, F.M. Mueller, P.G. van Engen, and K.H.J. Buschow, Phys. Rev. Lett. 50, 2024 (1983).

${ }^{2}$ R.J. Soulen, Jr., J.M. Byers, M.S. Osofsky, B. Nadgorny, T. Ambrose, S.F. Cheng, P.R. Broussard, C.T. Tanaka, J. Nowak, J.S. Moodera, A. Barry, and J.M.D. Coey, Science 282, 85 (1998).

${ }^{3}$ H. Ohno, Science 281, 951 (1998); F. Matsukura, H. Ohno, A. Shen, and Y. Sugawara, Phys. Rev. B 57, R2037 (1998).

${ }^{4}$ H. Akinaga, T. Manago, and M. Shirai, Jpn. J. Appl. Phys. 39, L1118 (2000).

${ }^{5}$ M. Shirai, Physica E (Amsterdam) 10, 143 (2001); S. Sanvito and N.A. Hill, Phys. Rev. B 62, 15553 (2000); A. Continenza, S. Picozzi, W.T. Geng, and A.J. Freeman, ibid. 64, 085204 (2001); I. Galanakis, ibid. 66, 012406 (2002); W.-H. Xie, Y.Q. Xu, B.-G. Liu, and D.G. Pettifor, Phys. Rev. Lett. 91, 037204 (2003).

${ }^{6}$ B.G. Liu, Phys. Rev. B 67, 172411 (2003).

${ }^{7}$ I. Galanakis and Ph. Mavropoulos, Phys. Rev. B 67, 104417 (2003)

${ }^{8}$ I. Galanakis, P.H. Dederichs, and N. Papanikolaou, Phys. Rev. B 66, 134428 (2002); I. Galanakis, P.H. Dederichs, and N. Papanikolaou, ibid. 66, 174429 (2002).

${ }^{9}$ S.A. Wolf, D.D. Awschalom, R.A. Buhrman, J.M. Daughton, S. von Molnar, M.L. Roukes, A.Y. Chtchelkanova, and D.M. Treger, Science 294, 1488 (2001).

${ }^{10}$ H. Ebert and G. Schütz, J. Appl. Phys. 69, 4627 (1991).

${ }^{11}$ V. Yu Irkhin and M.I. Katsnelson, Usp. Fiz. Nauk 164, 705 (1994) [Phys. Usp. 37, 659 (1994)]; V. Yu Irkhin and M.I. Katsnelson, J. Phys.: Condens. Matter 2, 7151 (1990); L. Chioncel, M.I. Katsnelson, R.A. de Groot, and A.I. Lichtenstein, Phys. Rev. B 68, 144425 (2003).

${ }^{12}$ N. Papanikolaou, R. Zeller, and P.H. Dederichs, J. Phys.: Condens. Matter 14, 2799 (2002); H. Ebert, in Electronic Structure and Physical Properties of Solids, Lecture Notes in Physics Vol.
535, edited by H. Dreyssé (Springer, Berlin, 2000), p. 191; H. Ebert, B. Drittler, and H. Akai, J. Magn. Magn. Mater. 104-107, 733 (1992).

${ }^{13}$ D.D. Koelling and B.N. Harmon, J. Phys. C 10, 3107 (1977).

${ }^{14}$ L.J. Gray and T. Kaplan, J. Phys. A 86, 1555 (1986).

${ }^{15}$ J.H. Zhao, F. Matsukura, K. Takamura, E. Abe, D. Chiba, and H. Ohno, Appl. Phys. Lett. 79, 2776 (2001).

${ }^{16}$ N. Papanikolaou, N. Stefanou, P.H. Dederichs, S. Geier, and G. Bergmann, Phys. Rev. Lett. 69, 2110 (1992).

${ }^{17}$ Y.-Q. Xu, B.-G. Liu, and D.G. Pettifor, Phys. Rev. B 66, 184435 (2002).

${ }^{18}$ Within the LDA, the Mn $d$ states can be high in energy, close to $E_{F}$, when the double-exchange mechanism dominates [H. Akai, Phys. Rev. Lett. 81, 3002 (1998); K. Sato, P.H. Dederichs, and H. Katayama-Yoshida, Europhys. Lett. 61, 403 (2003)]. But within the $\mathrm{LDA}+U$ the $d$ states can be much lower, pushing up the As $p$ states and the valence-band [K. Sato, P.H. Dederichs, H. Katayama-Yoshida, and J. Kudrnovský (unpublished)]. This is suitable for investigation within $p-d$ Hamiltonian methods [T. Dietl et al., Science 287, 1019 (2000)]. In any case, (Ga, Mn) As can be considered a ferromagnetic semiconductor, the properties of which (Curie temperature, resistivity, etc.) are sensitive to further doping or on defects such as antisites or interstitials.

${ }^{19}$ Peter Y. Yu and Manuel Cardona, Fundamentals of Semiconductors: Physics and Materials Properties, 3rd ed. (Springer-Verlag, Berlin, 2001).

${ }^{20}$ T. Dietl, H. Ohno, and F. Matsukura, Phys. Rev. B 63, 195205 (2001).

${ }^{21}$ J.G. Braden, J.S. Parker, P. Xiong, S.H. Chun, and N. Samarth, Phys. Rev. Lett. 91, 056602 (2003).

${ }^{22}$ K.E.H.M. Hanssen, P.E. Mijnarends, L.P.L.M. Rabou, and K.H.J. Buschow, Phys. Rev. B 42, 1533 (1990). 